\title{
Noninvasive Extraction of Dynamic Information of Human Vascular Wall
}

\author{
Ping Gan Hongming Tan Lili Chen Jiao Feng \\ Laboratory of Biomedical Engineering, Chongqing Medical University, Chongqing 400016, P.R.China
}

\begin{abstract}
Many cardiovascular diseases such as hypertension, artherosclerosi are subject to remodel the characteristics of vascular wall; at the same time, this change is able to result in a counteractive with these diseases. It is significant to find out vascular pathological changes as early as possible. In this paper, a new conception, pressure displacement wave of vascular wall (PDWVW), and a novel method that is used to diagnose the vascular disease initially were proposed for the first time based on the principle of Frank elasticity of vascular wall, developed an original diagnostic equipment -noninvasive diagnostic system of human vascular wall, and validated the feasibility and practicability of the method and equipment through clinical experiments.
\end{abstract}

Keywords: Vascular wall, Noninvasive measurement, Diagnostic system

\section{Introduction}

Many researches have demonstrated that the occurrence of cardiovascular diseases has close correlation with elasticity and geometrical conformation of human vascular. Once a cardiovascular disease arises, it is certain that the vascular geometrical conformation and mechanical elasticity should have been altered [1]. However, those diseases occurred, such as hypertensions, artherosclerosi, only are the signs or results of vascular wall's pathological changes. In current clinical diagnoses, it is still unsolvable that how to find and diagnose the alteration of vascular geometrical conformation and mechanical elasticity as early as possible [2][3]. The existing noninvasive diagnostic methods of vascular wall (Doppler ultrasound, CT, MRI) have many disadvantages, such as the complexity of operation, highly cost, and poor accuracy. The more important thing is that all these methods existed only may diagnose vascular diseases qualitatively not from its function, but from its modality [4].

In principle, human vascular wall, which is flexible and elastic, is a cavum tissue; it is able to obtain abundant information which is used to assure function of vascular wall when vascular wall is under the pressure stimulation from both inside and outside of it. In this paper, the conception of pressure displacement wave of vascular wall (PDWVW) is proposed for the first time [5]. Moreover, a novel method that can diagnose cardiovascular disease early was developed based on the PDWVW theory, and designed noninvasive dynamic information computer measurement system of vascular wall. The validity of DPWVW conception and applicability of the measurement system have been proved through clinical experiments in cooperation with the first affiliated hospital of Chongqing medical university.

\section{Noninvasive dynamic information of vascular wall}

Noninvasive dynamic information of vascular wall integrate multiple medical information parameters which can be obtained using noninvasive measurement techniques under special condition and have tight connection with the motion and elastic state of vascular wall. Because the correlation between the motion state of vascular wall and the heart's movement is quite close, exactest parameter that reflects the function of circulation system should be the noninvasive dynamic information of vascular wall.

In order to distinguish the noninvasive dynamic information of vascular wall from other parameters existed, such as pulse wave, pressure wave, and diameter wave and so on[6], here author firstly brought forward and defined a new conception - pressure displacement wave of vascular wall (PDWVW), which is the real-time information acquired using noninvasive measurement technique that is the displacement shift of vascular wall $\mathrm{X}(\mathrm{t})$ following the movement of time under outside pressure in light of the changes of special regularity (perhaps linear descending). 
In previous researches [5], author demonstrated that it is easy to acquire a special pressure signal if a high sensitive and accurate sensor is put on the brachial artery and radial artery in the process of conventional measurement of blood pressure that the pressure will be stable alteration according to linear descending. The signal is the PDWVW defined previously, which mixed up multiple useful information that can exhibit the function and modality of vascular wall and circulation system.

Moreover, it is quite apparent that PDWVW is keeping accelerating motion from the results of measurement, which means that it generated velocity and acceleration. PDWVW will vibrate subtly along with systolic and diastolic period. So, noninvasive dynamic information of vascular wall defined here not only denotes PDWVW but also contains the velocity and acceleration of PDWVW, volume wave of finger and so on. Otherwise, in order to improve the accuracy of the noninvasive dynamic information measurement system of vascular wall, electrocardiogram signal and heart beat sound signal was used to be basic standard that is able to compare to PDWVW, and its velocity and acceleration.

\section{Hardware structure of the noninvasive measurement system}

Hardware of this system consists of three parts which include input part, amplified unit and $\mathrm{A} / \mathrm{D} \mathrm{C}$ part (Fig.1).

\subsection{The input part}

A semiconductor Piezoresistiv pressure microsensor (static electricity capacitive pressure micorsensor HEM-423C), which has excellent performance such as good stability and repetition, tiny affected by distribution and parasitic capacity, low power consumption, good nonlinearity ,high accuracy, nice frequency response, strong anti-intervention, small volume and so on compare with other sensors, and completely is eligible to this system, was employed to collect signals from outer pressure of human body.

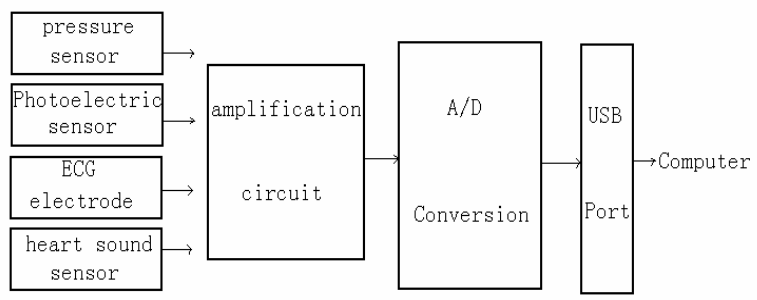

Fig.1: The schematic diagram of system.
An infra-red transmission Photoelectric pulse wave microsensor (wavelength is $700-860 \mathrm{~nm}$ ) was used to acquire the volume wave of forefinger or middle finger terminal vas. The drift of testing curve is easy to be banned using the infra-red photoelectric sensor that has good low frequency responses, high accuracy, wide rang of frequency response, strong anti-intervention. Either pulse wave or vas volume wave is able to be measured by it, and has excellent accuracy and linearity.

In addition, a heart beat sound sensor that has been refitted from average stethoscope was hired to collect the heart sound signal.

\subsection{Amplified unit}

All bio-signals on human body that could be collected have characteristics in common which include high resistance, slight signal, low frequency; the more important thing is that they are involved with many noises. In amplified unit, special methods were taken to avoid these defects previous mentioned. The analog circuit that is used to acquire the physical single must be high input impedance and common-mode rejection ratio. Furthermore, it should have large gain and broad bandwidth in accordance with amplitude of different physical signal.

\subsubsection{Preamplifier part}

The preamplifier circuit made use of low noise amplifier and decrease the input noise as much as possible so that it can optimize the signal-to-noise ratio. A high accurate instrumentation amplifier AD620, which was made by Analog Devices company, and has low power consumption, high accuracy and common-mode rejection ratio, temperature drift inhibit, was employed in this system. Its maximum gain can be as large as 10000 times, 93dB CMRR, $800 \mathrm{KHz}$ bandwidth.

\subsection{2 low-pass filter}

The amplified circuit has crucial impact to the success of this system, which means that its performance can affect whole system. The basic trait of physical signal is that they are low signal-to noise ratio and amplitude. $\mathrm{RC}$ low-pass filter amplifier was designed in the system, which was made up of TL084. The passband cut-off frequency of low-pass filter that can be used to impair effectively intervention of high-order harmonic and background noise is that $f_{p}=1 / 2 \pi R C=300 \mathrm{~Hz}$. The $50 \mathrm{~Hz}$ electromagnetic interference was removed through a double-T filter, and a follower, which was employed to enhance the input impedance of physical signal that it will make the useful signal pass to the next part without any loss, was connected behind the double-T filter.

3.2.3 Post amplifier part 
In order to clearly and truly reflect the alteration process of blood flow signal ,a differential and integral circuit was designed to get the pressure displacement wave signal and blood volume wave following the locomotion of time that will improve the stability of this system. Otherwise, a resistance and capacitance was in series to strength the capacity of anti-interference and inhibit the self excited oscillation. A capacitance was added with the feedback resistance as phrase compensation.

\subsection{Analog/Digital Conversion(A/DC)}

An excellent data acquisition card (AD8223), which has 32-channels USB interface, and can complete high-speed dynamic data acquisition, was used in this system (Fig 2). Multiple-channelss physical signals were inputted through port $\mathrm{J} 3$, and entered into the amplifiers via multiple-switch which will achieve optimal impedance matching, then the signal would get into programmed-controlled amplifier; A/DC would be completed in A/D chip.

Single Chip Micyoco (SCM) is able to execute Integrated functions which will choose the corresponding channel matched different signals, then trigger and transform $\mathrm{A} / \mathrm{D}$ to FIFO that can be detected by USB bus; lastly it will be input to PC.

Transformation resolution of the card is 12 Bit with $4 \mathrm{~K}$ cache. Its sampling frequency reached to $100 \mathrm{KHz}$, accuracy and error of sampling, respectively, is $12 \mathrm{Bit,}$ 1LSB.

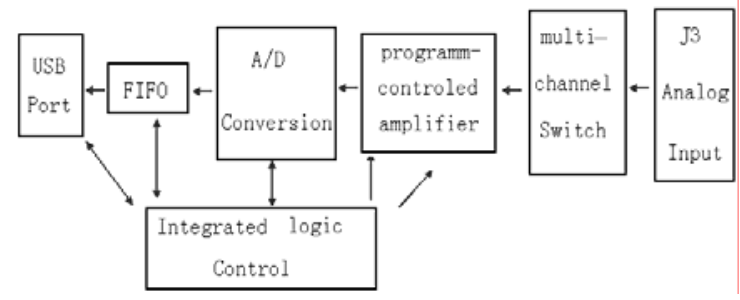

Fig.2: The principle diagram of acquisition card.

\section{The procedure of measurement and structure of software}

\subsection{The procedure of measurement}

(1) Examine patients carefully before measurement, including age gender weight height case history and the cardiovascular function, record these with table.

(2) Let patients lie down on bed peacefully about 5-10 min, relax them.

(3) Place the binding belt of sphygmomanometer on the radial artery, a Piezoresistiv microsensor that is hired here to collect the special wave- PDWVW was put on the radial artery far from ancon about $3 \mathrm{~cm}$.

(4) Place the transmission photoelectric microsensor on the terminals of forefinger or middle finger acquired the volume wave of vascular wall as the same hand as previous.

(5) To avoid the influence of inter-electrode capacitance, rub the position that the electrodes of ECG standard II lead will be placed using absolute alcohol.

(6) Place the heart sound sensor on the ventricular apical of $5^{\text {th }}$ rib in left-middle line to measure signal.

(7) Power on the system that it will execute systematical test automatically; firstly check out whether the output signals of ECG、volume wave、 heart sound are normal; Secondly inflate the binding belt of sphygmomanometer to augment stress up to $30 \mathrm{mmhg}$, then deflate the binding belt in light of the linear descending regularity, acquire these signals, and real-time manifest on screen (Fig 3).

\subsection{The structure of software}

Data acquisition program of this system was designed with $\mathrm{C}^{++}$6.0. The computer interface take advantage of the interrupt method which means it needs an interrupt service program to operate; in addition to application program, an extra interrupt program is indispensable. To ensure the sample-and-hold amplifier keep working after starting $3 \mu \mathrm{s}$, the outside trigger method was used in this system. At application program, it will get data from interrupt program

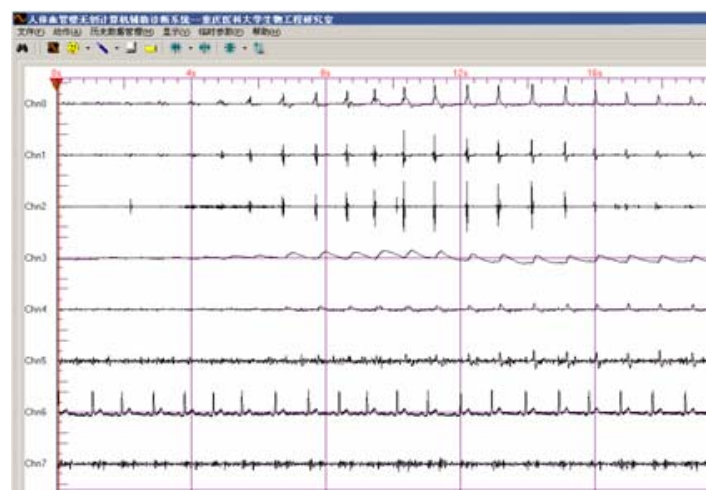

Fig.3: Output of wave form.

Using timber; the data acquired that value is less than 15 denotes it is not true data but frame synchronization. The real waveform the system collected had shown in Fig 3. The scale on top of screen is the time base, unit is $\mathrm{S}$. channel 0 is the special waveform the system can measure - PDWVW; channel 1and channel 2 is, 
respectively, velocity and acceleration waveform of PDWVW; channel 3 is the volume waveform; channel 4 and channel 5 is, respectively, velocity and acceleration waveform of volume waveform; channel 6 is ECG signal; channel 7 is cordis sound signal.

\section{Results and discussion}

The validity of DPWVW conception and feasibility of noninvasive diagnostic system of human vascular wall have been proved through clinical experiments in cooperation with the first affiliated hospital of Chongqing medical university.

(1) The status of whole cardiovascular system can be manifested quantitatively by the system from the real-time waveform measured in Fig 3.

(2) Not only inside dynamic information (the volume waveform and its velocity 、 acceleration, ECG、 cordis sound ) of vascular wall but also outside information(PDWVW and its velocity 、 acceleration) of vascular wall were extracted, and can be real-time come out with 8 channels. The accuracy of this system is far higher than current methods existed.

(3) In Fig 4, the PDWVW between healthy individual and patient was compared. The difference may be judged easily from Fig 4(a) and Fig 4(b). In addition, the PDWVW measured by the system can diagnose patients with premature ventricular contraction. Fig 5 is the PDWVW (Ch0) of patients with premature ventricular contraction, the synchronous ECG (Ch6) proved this diagnoses.

(4) It is quite obvious the data used to diagnose disease is acquired from both inside and outside of vascular wall which is an excellent method to early noninvasive measure cardiovascular disease is more accurate and useful than those data only extracted from one side. Author will specifically analyze the characteristics and clinical meaning of each waveform collected by the system in the coming articles.

\section{Conclusions}

To diagnose cardiovascular diseases on the initial phase has became more and more emergent and important since this kind of disease has been No.1 factors that threaten the health of human being.

In this paper, author proposed a new notion PDWVW for the first time, and developed a novel measurement system.

The experiment's results have demonstrated that this new notion is correct and valid. Moreover, the diagnostic system that was developed based on this new notion can find out vascular disease not only from its function, but also from its modality.

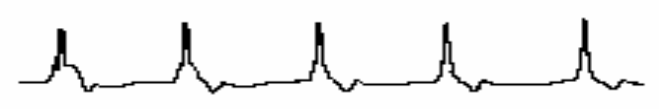

(a)

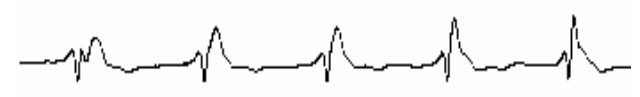

(b)

Fig.4: PDWVW of healthy individual and patient with hypertension.(a) PDWVW of healthy individual;(b) PDWVW of patient with hypertension.

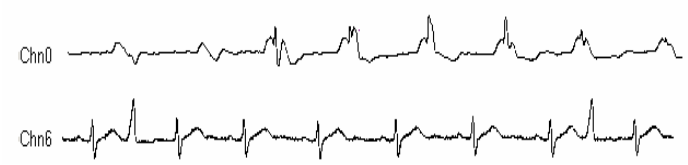

Fig.5: PDWVW of patient with premature ventricular contraction.

\section{Acknowledgement}

This work is supported by Science \& Technology Commission of Chonqing, China(Grant No. CSTC 2005BB2213).

\section{References}

[1] L.D. Jou, Numerical Simulation of the Flow in the Carotid Bifurcation, Theoret. Comput Fluid Dynamics, 10:239-248, 1998.

[2] W.Z. Zhang, Study on the arterial elastidty function in the Chinese healthy subjects, Chin.J.Cardiol , 4:245-249, 2003.

[3] A.K. Qiao, S .G. Wu, The theory of pulse wave in atery, J Biomed Eng, 17(1):95-100, 2000.

[4] A.G.. Ventura, S. Reisman, A study of factors affecting cardiovas - cular reactivity, Proceedings of the IEEE 26th Annual North-east Bioengineering conference. (Cat.No.00CH37114), PP.153-54, 2000.

[5] P. Gan, G.H. Hu, Research on collecting method for dynamic information of vascular wall, Transducer and Microsystem Technologies, 25(3):18-21, 2006.

[6] A. Roland, H.Y. Wang, Clinical applications of arterial stiffness and pulse wave velocity, Bejing: People’s Military Medical Press,PP.1-13, 2005. 
[7] L.C. Chen, G.H. Hu, Counting-type Analog Quantity and Development of Mutli-channel

Telemetering Computer System for Dynamic Information about Human Vascular Wall, $J$ Biomed Eng, 23(1):200-204, 2006. 